\title{
Por qué usar preferentemente stents no farmacoactivos
}

\section{Why it is preferable to use non-drug-eluting stents}

\author{
Dr. Manuel Gómez Recio ${ }^{\bowtie}$ y Dra. Rosa Lázaro García
}

Servicio de Cardiología. Unidad de Hemodinámica. Complejo Hospitalario Torrecárdenas. Almería, España.

Full English text of this article is also available

INFORMACIÓN DEL ARTÍCULO

Palabras clave: Stent convencionales, Stent farmacoactivo, Angioplastia coronaria Key words: Bare metal stents, Drug-eluting stents, Coronary angioplasty

Desde la irrupción en Europa, en 2002, de los stents farmacoactivos (SFA) de primera generación (la FDA siglas en inglés de la Administración de Alimentos y Medicamentos- no aprobó su uso hasta 2004), su utilización fue rápidamente creciente en España y en 2005 superaba el $50 \%$ del total de stents implantados (41.352 SFA vs. 39.217 convencionales, registro de la Sección de Hemodinámica). Según este propio registro se ha mantenido un crecimiento continuo hasta el 2011, año en el que se implantaron 58.211 SFA vs. 36.490 convencionales $(61,47 \%)$. Hasta este mismo año (último registro del que tenemos datos) ${ }^{1}$ se han implantado 428.748 SFA en España; sin embargo, las variaciones de uso entre hospitales y su traducción en las autonomías, que son las que financian la sanidad, es enorme (Figura).

De igual manera, en 2011, en el País Vasco, el $81,49 \%$ de los stents que se usaron fueron SFA y en Galicia, 49,08\%. Andalucía se mantiene levemente por

$\triangle$ M Gómez Recio Hospital Torrecárdenas Servicio de Cardiología, Unidad de Hemodinámica Paraje Torrecárdenas s/n, CP 04009

Almería, España.

Correo electrónico: mgrecio@gmail.com debajo de la media (58,77 \%), pero en el Hospital Torrecárdenas sólo se llega al 17,96 \%. La variabilidad en cualquier aspecto de la práctica médica obliga a su análisis cuidadoso.

Existe un gran número de estudios publicados con las bondades de los SFA, en general financiados por las casas comerciales con objeto de conseguir la aprobación de su uso por las agencias (FDA o mercado europeo). A continuación se hace referencia a los datos de algunos registros, como el de la Sección de Hemodinámica o, de gran peso, como el registro sueco (SCAAR).

En 2007, el primer análisis del registro sueco demostraba que el uso de SFA se asociaba con una tasa mayor de mortalidad que con los stents convencionales $^{2}$. Sin embargo, en nuestro entorno, el predominio del uso de SFA de primera generación era imparable. No era el único estudio que daba la voz de alarma ${ }^{3}$, las guías del NICE (The National Institute for Health and Clinical Excellence) del Sistema Nacional de Salud británico ${ }^{4}$ limitaban el uso de los SFA a las lesiones tratadas de menos de $3 \mathrm{~mm}$ de calibre o de longitud mayor de $15 \mathrm{~mm}$, y cuando la diferencia de precio entre el SFA y el convencional era menor de 300,00 $£$ (unos $345,00 €)$. A pesar de ello la tendencia de uso del SFA siguió una curva ascendente, probablemente debido a 


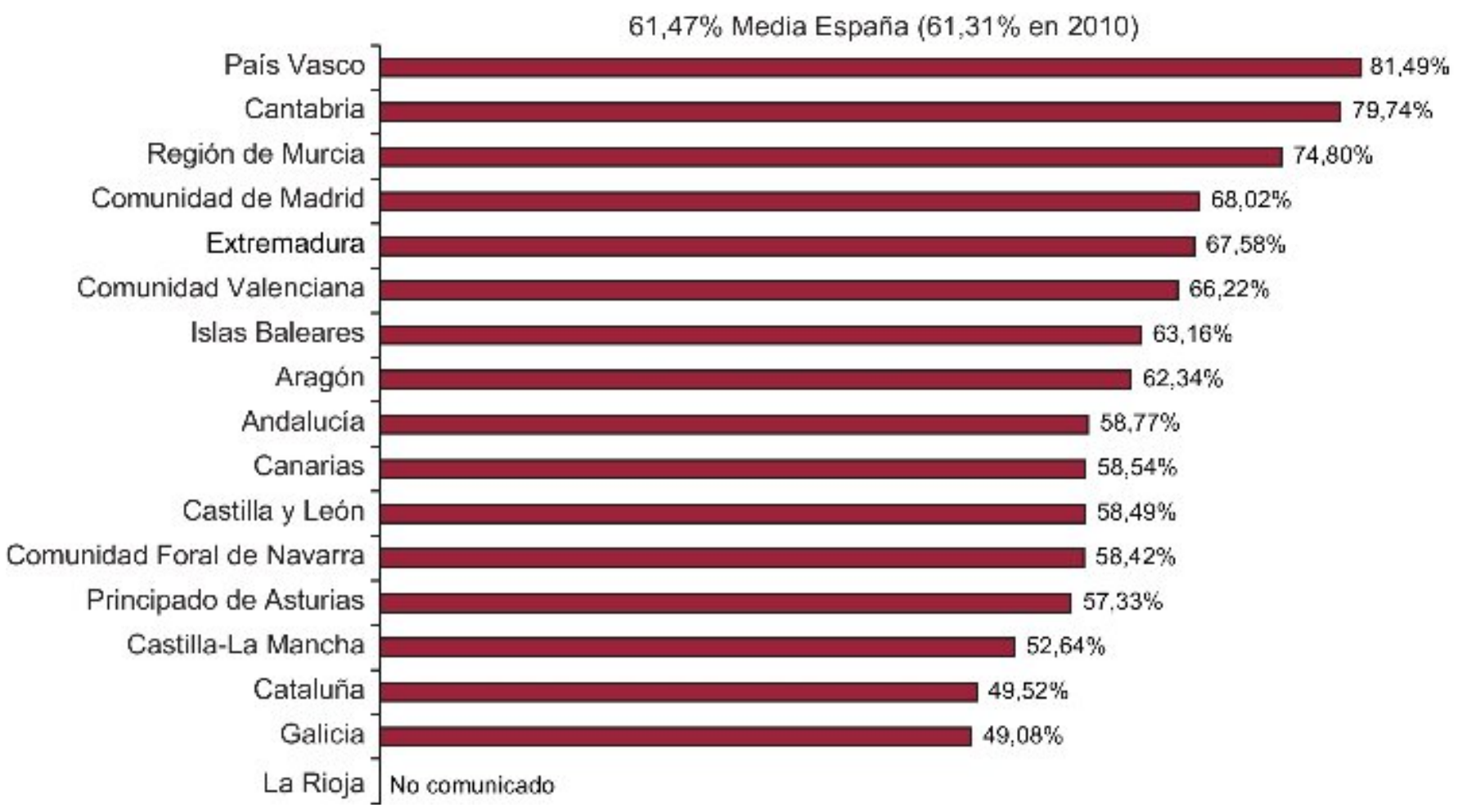

Figura. Porcentaje de SFA respecto al total de unidades de stent implantadas según la comunidad autónoma de España.

Tomado de DíazJ F, et al. ${ }^{\mathbf{1}}$ con permiso de Revista Española de Cardiología.

la influencia del aluvión de estudios acerca de sus bondades (a veces con tasas nulas de sucesos desfavorables), apoyado por estrategias comerciales que mostraban un dispositivo nuevo y eficaz contra lo que había sido señalado como el principal problema, la reestenosis. Además, las primeras generaciones de SFA tenían plataformas de navegabilidad, flexibilidad y por tanto, una seguridad de uso claramente inferior a los stents convencionales (sin que se pueda encontrar ninguna referencia en la literatura).

Los SFA han demostrado una única ventaja sobre los convencionales: la reducción de la tasa de reestenosis. En el primer registro SCAAR estas tasas de reestenosis eran de 4,5 vs. 5,5\%, respectivamente ${ }^{2}$. Sin embargo, cuando se analizan los estudios diseñados para la aprobación de los distintos dispositivos las cifras son diferentes: en la publicación del estudio RAVEL en $2002^{5}$, se comparó el stent liberador de sirolimus con el convencional, la tasa de reestenosis era de 0 vs. $27 \%$. Sorprendentemente la tasa de trombosis del stent era $0 \%$ en ambas ramas.

Los talones de Aquiles de los SFA son esencialmente dos:

- Seguridad: el riesgo de trombosis tardía y muy tar- día asociada a los SFA se publicó inmediatamente ${ }^{3}$ y por tanto, la necesidad de mantener doble antiagregación durante un tiempo indefinido. Se han publicado, y hemos presenciado, casos de trombosis de SFA de primera generación a los 6 y 7 años de su implantación. Este problema es el que explica la mayor mortalidad en los registros iniciales con este tipo de stent, respecto a la angioplastia con stent convencional.

- Coste: Las diferencias de precio entre la angioplastia con balón o con stent convencional, inicialmente abismales (en España llegaron a ser de $2.000 €$ en la época del monopolio de la primera casa comercial), hacían impensable que el beneficio marginal generado por la reducción de la reestenosis hiciese razonable el incremento de coste del uso indiscriminado de SFA.

Sin embargo, en todos los estudios en los que se compara algo que no sea la certificación de uso del stent por las agencias estatales, por ejemplo en la comparación de angioplastia con la cirugía de revascularización en distintos subgrupos, los objetivos importantes de resultados (mortalidad, infarto de miocardio, 
accidente cerebrovascular) no difieren cuando se ha comparado la cirugía con angioplastia con globo, stent convencional, o SFA (estudios FREEDOM, BARI, ARTS) ${ }^{6}$. Quiere decir que no existe ningún dilema ético, en el que la seguridad del paciente está en juego. Se ha contraargumentado que la reestenosis no es un fenómeno banal y se ha dicho que, incluso en hasta un 20 $\%$ de los casos, produce infarto de miocardio ${ }^{7}$. Todo ello para equiparar los objetivos (endpoints en su idioma original) importantes en los análisis comparativos. Es difícil entender en la práctica clínica por un lado la variable definición de infarto que se usa en función de lo que se quiere demostrar, y por otro lado, no mostrar consternación por el sistema de cuidado y seguimiento de quien en sus series tiene tal porcentaje de infartos como clínica de debut de una reestenosis.

Además, el beneficio marginal en esta situación no traduce un cambio sustancial en la actividad de Cardiología Intervencionista en este escenario, pues en el registro de la Sección de Hemodinámica el número de intervenciones en lesiones reestenóticas ha seguido aumentando año tras año, con estabilización en 2010 y 2011, a pesar del uso masivo de SFA desde el 2005.

En síntesis, el coste diferencial del uso de los SFA respecto a los convencionales ha significado en nuestro país, al menos, 750 millones de euros en la última década. En el Hospital de Torrecárdenas, donde empezó la actividad de Cardiología Intervencionista en el 2004, se han realizado desde entonces más de 5.500 ACTP, de ahí que se hayan implantado más de 8.000 stents. Si se hubieran utilizado SFA en el porcentaje de la media española $(61,5 \%)$ en lugar del $18 \%$ de este hospital, se hubiesen gastado 4 millones y medio de euros más. Sin embargo, nuestros resultados en salud no son diferentes de los conseguidos en centros o comunidades autónomas, que usan indiscriminadamente los SFA. Nuestra actividad en el tratamiento de la reestenosis se ha mantenido año tras año alrededor del $5 \%$.

Si se ajustan las indicaciones de uso de los SFA a su verdadera utilidad, que es reducir la reestenosis en las lesiones con alto riesgo reestenótico, el porcentaje de uso no fuera superior al $30 \%$ (registro SCAAR).

En nuestro centro la indicación de uso de SFA se puede resumir en, alto riesgo de reestenosis en lesiones cuya reestenosis tenga trascendencia clínica:

- Lesiones largas en vasos menores de $3 \mathrm{~mm}$ de diámetro, especialmente en pacientes diabéticos.
- Lesiones reestenóticas.

- Oclusiones crónicas.

- Gran territorio miocárdico en riesgo.

No se puede olvidar que la angioplastia, en la enfermedad coronaria crónica, mejora la calidad de vida al controlar los síntomas, pero no cambia la supervivencia.

\section{Infarto agudo de miocardio}

La angioplastia primaria constituye una parte cada vez más importante de nuestra actividad, tanto numéricamente como porque es la actuación de Cardiología Intervencionista que más influye en la supervivencia. Los estudios recientes sugieren que no hay ninguna ventaja en el uso de SFA en esta indicación ${ }^{8}$. Por otra parte, se propone que en muchos casos se pueda evitar incluso la implantación de stent tras tromboaspiración, lo que evita el grave problema del fenómeno de no reflujo tras la implantación de stent ${ }^{9,10}$. Otra opción, en este caso, es el uso de stents convencionales cubiertos con micromalla que evitan la migración del trombo con unos resultados excelentes ${ }^{11}$.

\section{Situación actual}

Felizmente los SFA de primera generación han sido sustituidos por otros más seguros. En la revisión más reciente del $\operatorname{SCAAR}^{12}$ la tasa de revascularización por reestenosis al año fue de 4,6 \% en el grupo de stent convencional, de $3,1 \%$ en el de SFA de primera generación y de 2,2 \% en los SFA actuales. Más importante, la tasa de trombosis intrastent comprobada a los 2 años pasa de $1,3 \%$ con los SFA antiguos a $0,6 \%$ en los actuales. En los datos del SCAAR publicados en PLOS Medicine en febrero de $2013^{13}$, donde se analiza la técnica de implantación (presión de despliegue del stent y post-dilatación) en 93.692 stents implantados (69 \% convencionales) desde 2008 y seguidos durante 2 años, la tasa global de reestenosis fue de 5,09 \% y la de trombosis, de 1,07\%, principalmente influidas por la presión de implantación (mínima trombosis con 2021 atmósferas y más reestenosis con post-dilatación).

Por tanto, los nuevos stents, tanto convencionales, de distintas aleaciones y distintos recubrimientos pasivos, como farmacoactivos, son cada vez mejores, más seguros, de más fácil implantación y con mejores resultados a largo plazo.

Depurar las técnicas de implantación es cada vez más crítico para el resultado. Mantener nuestras prác- 
ticas actualizadas y seguras es el reto diario. Y como ha demostrado esta saga de los SFA, hay que ser muy crítico con las novedades tecnológicas y controlar constantemente nuestros resultados.

\section{REFERENCIAS BIBLIOGRÁFICAS}

1. Díaz JF, de La Torre JM, Sabaté M, Goicolea J. Registro español de Hemodinámica y Cardiología Intervencionista. XXI Informe Oficial de la Sección de Hemodinámica y Cardiología Intervencionista de la Sociedad Española de Cardiología (1990-2011). Rev Esp Cardiol. 2012;65:1106-16.

2. Lagerqvist B, James SK, Stenestrand U, Lindbäck J, Nilsson T, Wallentin $\mathrm{L}$, et al. Long-term outcomes with drug-eluting stents versus bare-metal stents in sweden. N Engl J Med. 2007;356(10):1009-19.

3. Virmani R, Guagliumi G, Farb A, Musumeci G, Grieco N, Motta $\mathrm{T}$, et al. Localized hypersensitivity and late coronary thrombosis secondary to a sirolimuseluting stent. Circulation. 2004;109(6):701-5.

4. Drug-eluting stents for the treatment of coronary artery disease. NICE technology appraisal guidance 152. Part review of NICE technology appraisal guidance 71. Review date: April 2009. [Artículo en Internet]. 2009. [citado 01 mar 2013]. Disponible en:

http://www.nice.org.uk/nicemedia/pdf/TA152Guid ance.pdf

5. Morice MC, Serruys PW, Sousa JE, Fajadet J, Ban Hayashi $\mathrm{E}$, Perin $\mathrm{M}$, et al. A randomized comparison of asirolimus-eluting stent with a standard stent for coronary revascularization. N Engl J Med. 2002; 346(23):1773-80.

6. Farkouh ME, Domanski M, Sleeper LA, Siami FS, Dangas $G$, Mack $M$, et al. Strategies for multivessel revascularization in patients with diabetes. $N$ Engl J Med. 2012;367(25):2375-84.
7. Stefanini GG, Holmes DR. Drug-eluting coronaryartery stents. N Engl J Med. 2013; 368(3):254-65.

8. Kalesan B, Pilgrim T, Heinimann K, Räber L, Stefanini GG, Valgimigli $M$, et al. Comparison of drugeluting stents with bare metal stents in patients with ST-segment elevation myocardial infarction. Eur Heart J. 2012;33(8):977-87.

9. Kelbaek H, Engstrøm T, Ahtarovski, Lønborg J, Vejlstrup N, Pedersen F, et al. Deferred stent implantation in patients with ST-segment elevation myocardial infarction: a pilot study. Eurolntervention. 2013;8(10):1126-33.

10.Escaned J, Echavarría-Pinto M, Gorgadze T, Gonzalo $\mathrm{N}$, Armengol F, Hernández R, et al. Safety of lone thrombus aspiration without concomitant coronary stenting in selected patients with acute myocardial infarction. Eurolntervention. 2013;8(10):1149-56.

11.Costa JR, Abizaid A, Dudek D, Silber S, Leon MB, Stone GW. Rationale and design of the MGuard for acute ST elevation reperfusion (MASTER) Trial. 2013;82(2):184-90.

12.Sarno G, Lagerqvist B, Fröbert O, Nilsson J, Olivecrona $\mathrm{G}$, Omerovic $\mathrm{E}$, et al. Lower risk of stent thrombosis and restenosis with unrestricted use of 'new-generation' drug-eluting stents: a report from the nationwide Swedish Coronary Angiography and Angioplasty Registry (SCAAR). Eur Heart J. 2012; 33(5):606-13.

13. Fröbert O, Sarno G, James SK, Saleh N, Lagerqvist B. Effect of stent inflation pressure and post-dilatation on the outcome of coronary artery intervention. A Report of more than 90.000 stent implantations. PLOS ONE [Internet]. 2013 [citado 18 Jul 2013]; 8(2):e56348. Disponible en: http://www.ncbi.nlm.nih.gov/pmc/articles/PMC35 71959/ 